\title{
The Effect of Pure State Structure on Nonequilibrium Dynamics
}

\author{
C.M. Newman \\ newman @ cims.nyu.edu \\ Courant Institute of Mathematical Sciences \\ New York University \\ New York, NY 10012, USA \\ D.L. Stein \\ daniel.stein @ nyu.edu \\ Dept. of Physics and Courant Institute of Mathematical Sciences \\ New York University \\ New York, NY 10012, USA
}

\begin{abstract}
Motivated by short-range Ising spin glasses, we review some rigorous results and their consequences for the relation between the number/nature of equilibrium pure states and nonequilibrium dynamics. Two of the consequences for spin glass dynamics following a deep quench to a temperature with broken spin flip symmetry are: (1) Almost all initial configurations lie on the boundary between the basins of attraction of multiple pure states. (2) Unless there are uncountably many pure states with almost all pairs having zero overlap, there can be no equilibration to a pure state as time $t \rightarrow \infty$. We discuss the relevance of these results to the difficulty of equilibration of spin glasses. We also review some results concerning the "nature vs. nurture" problem of whether the large- $t$ behavior of both ferromagnets and spin glasses following a deep quench is determined more by the initial configuration or by the dynamics realization.
\end{abstract}

KEY WORDS: spin glass; nonequilibrium dynamics; deep quench; stochastic Ising model; broken ergodicity; coarsening; persistence; damage spreading. 


\section{Introduction}

Experimental signatures of laboratory spin glasses - irreversibility, history dependence, aging - demonstrate that these systems are out of equilibrium during the timescale of most, if not all, experimental measurements [1, 2, 3, 4, 15, 6, 7, 8, 9, 10, 11, 12, 13, 14, 15, 16, 17, 18, 19, 20]. This puts us in the unusual position of attempting to explain the nonequilibrium dynamics of a system whose equilibrium statistical mechanics have yet to be worked out, or even understood on the most basic qualitative level. We still don't know, for example, whether there exists a true equilibrium phase transition to a spin glass phase in any dimension in the usual short-range models that are believed to describe laboratory spin glasses (many of which aren't short-range at all. This leads to the question of how well short-range models do in fact represent them - but since none of the models are at all undersood, that question remains moot for now.) Supposing that there is a phase transition in short-range models, we don't know their low-temperature properties, such as pure state multiplicity and structure. We don't even know what their zero-temperature (i.e., ground state) properties look like. (It would be an overstatement, however, to say we don't know anything. Numerical work has provided some valuable insight [21, 22, 23, 24, 25, 26, 27, 28, 29, 30, 31, 32, 33, 34, 35], there exist several competing pictures for the spin glass phase [36, 37, 38, 39, 40, 41, 42, 43, 44, 45], and rigorous and nonrigorous work of the authors has effectively ruled out some scenarios [46]. But we clearly still have a long way to go.)

This lack of progress is partly responsible for the viewpoint advocated by some that the only physics of spin glasses really worth looking at is their nonequilibrium dynamics. Although this seems to us a bit premature given that we don't really know what the equilibrium properties look like, there's no question that even if we did have a better picture of the equilibrium thermodynamics, we could still be a long way from explaining the many experimental observations of spin glass behavior.

Related to this, but not quite the same, is the prevalent viewpoint that the nonequilibrium dynamics of spin glasses (or any system with many competing thermodynamic phases) is sharply separated from their equilibrium behavior, in particular, their possessing many pure states. The actual presence of many pure states may or may not exist in real spin glasses, but the possibility has created substantial excitement about these systems and spurred numerous theoretical and numerical investigations. The basic idea behind this viewpoint lies in the dynamical invariance of pure states. That is, dynamics takes a spin configuration belonging to a pure state to another in the same pure state, but never to a configuration in a different pure state; equivalently, pure states are separated by dynamically insurmountable barriers. It therefore seems initially reasonable to conclude that the number of equilibrium pure states 
is dynamically irrelevant: since dynamics always occurs within a single pure state, no matter how long the timescale, knowledge of the equilibrium pure state structure doesn't tell one very much about the nonequilibrium dynamics.

The main point of this paper is to convince the reader that this idea is incorrect, and that the two (equilibrium structure and nonequilibrium dynamics) are in fact very much interrelated. Of course, the context in which an experiment is done is crucial in any statement of this kind. To support the above claim, we will review earlier papers of the authors, and discuss some mathematically rigorous theorems there that demonstrate a deep interconnection between equilibrium pure state structure and nonequilibrium dynamics in one of the farthest-from-equilibrium situations that has been studied: a sudden quench from very high to low temperature. As we proceed, we'll show along the way that a number of other widely-held beliefs break down in this much studied situation. One of these is the common assumption that the union of the basins of attraction of all of the pure states fills up most of the available state space, and that the boundaries between the pure states are lower dimensional and thereby form a set of measure zero in the set of all spin configurations (in fact, the opposite will turn out to be true). Another is that if many pure states are present in an infinite system, time averages don't agree with Boltzmann averages (in principle and perhaps even in practice, they can agree).

Our approach is general, and covers both ordered and disordered, Ising and non-Ising systems, although we will usually focus our attention on nearest-neighbor Ising spin glasses for specificity. We make no a priori assumptions about the real-space or state-space structure of the low-temperature spin glass phase, but instead derive several general principles and then explore their consequences.

We emphasize that our discussion centers on pure states, not metastable states. Crudely put, metastable states are surrounded by barriers that remain of $O(1)$ irrespective of the size of the system, while pure states are surrounded by barriers that diverge in the thermodynamic limit. (More precise definitions can be found in [46], but in this paper we sacrifice some mathematical precision for readability.) Metastable states are often proposed as responsible for the anomalous dynamical behavior of spin glasses. While we have no argument with this, we question the usefulness of the usual practice of inserting metastability by hand, requiring a guess as to the structure (usually in state space) and nature of the metastable states. (In fact, much of this structure can be determined $a b$ initio - e.g., a rigorous discussion of metastability in spin glasses and disordered systems can be found in [47].)

To keep the paper reader-friendly, we will present theorems without their proofs, which can be found in the cited references. 


\section{Pure states, dynamics, and equilibration}

Although the preceding discussion uses familiar terms and notions, their actual meanings require some work to pin down. For example, what does equilibration following a deep quench mean in an infinite system? Can it occur on any finite timescale? What does it mean for a system to evolve, or settle into, or even to "spend all its time inside," a single pure state? In order to proceed, we need to clarify these notions.

For specificity,we will mostly, but not exclusively, consider the Edwards-Anderson (EA) Ising spin glass [48] in zero external magnetic field. Its Hamiltonian is given by:

$$
\mathcal{H}_{\mathcal{J}}=-\sum_{<x y>} J_{x y} \sigma_{x} \sigma_{y}
$$

where the sites $x, y \in \mathbf{Z}^{d}$ and the sum is taken over nearest neighbors only. The couplings $J_{x y}$ are independent random variables, whose common probability density is symmetric about zero; we let $\mathcal{J}$ denote a particular realization of the couplings.

We next need to specify the dynamics. We are interested in the experimental situation in which a spin glass dynamically evolves following a deep quench. We model this as the quench of an infinite system governed by the Hamiltonian (1) from infinite to low temperature. This is done by first choosing the initial (time $t=0$ ) spin configuration $\sigma^{0}$ from the infinitetemperature ensemble where the individual spins are independent random variables equally likely to be +1 or -1 . We then use the usual Glauber dynamics, which is easiest to describe at zero temperature; there, flips that are energy lowering occur with probability 1 , flips that are energy neutral (neither lowering nor rasing the energy) occur with probability $1 / 2$, and flips that are energy raising occur with probability 0 . The exact choice of spin flip rates plays no role in our analysis as long as detailed balance is satisfied, so we may take the rate of considering flips to be 1 . At nonzero temperature any dynamical rule consistent with detailed balance can be used, such as the usual Metropolis or heat bath dynamics.

At any temperature $T$, we denote by $\omega$ a given realization of the dynamics (i.e., of the order in which spins are chosen to determine whether they flip according to the dynamical rules, combined with the outcome of each of these trials). Any $\omega$ can be regarded as a collection of random times $\left(t_{x, i}: x \in \mathbf{Z}^{d}, i=1,2, \ldots\right)$, each specifying when a spin flip at site $x$ is considered (forming a Poisson process in time for each $x$ ) along with random numbers $u_{x, i}$ that determine if the flip is taken.

So there are three sources of randomness in the problem: the couplings, the initial spin configuration, and the dynamics. Specific realizations for any given dynamical run are denoted respectively by $\mathcal{J}, \sigma^{0}$, and $\omega$. All three are needed to determine $\sigma^{t}$, the spin configuration at time $t$. We always take $\mathcal{J}$ to be fixed during any single run, to correspond to 
experimental situations on laboratory timescales.

\subsection{Equilibrium states}

Consider now a finite volume $\Lambda_{L}$, say a cube, of linear size $L$ (which may be arbitrarily large) centered at the origin. For a given boundary condition (b.c.), the equilibrium finite-volume Boltzmann distribution is given at temperature $T$ by

$$
\rho_{\mathcal{J}, T}^{(L)}(\sigma)=Z_{\mathcal{J}, L, T}^{-1} \exp \left\{-\mathcal{H}_{\mathcal{J}, L}(\sigma) / k_{B} T\right\}
$$

where the finite-volume spin configurations $\sigma$ are restricted to those obeying the b.c. and the partition function $Z_{\mathcal{J}, L, T}$ is such that the sum of $\rho_{\mathcal{J}, T}^{(L)}$ over all spin configurations in $\Lambda_{L}$ yields one.

The quantity $\rho_{\mathcal{J}, T}^{(L)}(\sigma)$ is of course simply a probability measure: it describes at fixed $T$ the probability of a given spin configuration $\sigma^{(L)}$ obeying the specified boundary condition appearing within $\Lambda_{L}$. One is also interested in infinite-volume measures $\rho=\rho_{\mathcal{J}, T}(\sigma)$ that specify the probability of any spin configuration appearing within $\Lambda_{L^{\prime}}$, for every $L^{\prime}$; we call such a $\rho$ a Gibbs state. The infinite-volume limit of any convergent sequence of $\rho_{\mathcal{J}, T}^{(L)}(\sigma)^{\prime}$ 's with any set of b.c.'s (which can vary with $L$ ) specifies a Gibbs state; one or many Gibbs states may exist, depending on the system, temperature, and dimension. (The assertion sometimes made that such infinite-volume quantities may not exist or make sense for spin glasses is incorrect: it is easily shown that they do exist and that they govern the equilibrium behavior of spin glasses; we just don't happen to know what they look like.) Gibbs states may be pure or mixed; see Sect. 4.1 of [46] for definitions and a detailed discussion. (The term "pure state" used throughout this paper refers to these pure equilibrium states.)

\subsection{Dynamical states}

To discuss the questions asked at the beginning of this section in a meaningful way, we also need to specify a dynamical probability measure with which Gibbs states can be compared. Consider then the infinite-volume spin configuration $\sigma^{t}$ at time $t>0$. As already mentioned, $\sigma^{t}$, a dynamical Markov process, depends on $\mathcal{J}, \sigma^{0}$, and $\omega$, but for ease of notation this dependence will be suppressed. We define the dynamical probability measure $\nu_{t}(\sigma)$ as the distribution of $\sigma^{t}$ over the dynamics $\omega$ for fixed $\mathcal{J}$ and $\sigma^{0}$. That is, $\nu_{t}$ tells us the probability for each $L$ of finding a particular spin configuration $\sigma^{(L)}$ within $\Lambda_{L}$ at time $t$ for a given $\mathcal{J}$ and starting configuration $\sigma^{0}$. We will also sometimes consider a measure $\nu_{t, \tau}$ which is the distribution of $\sigma^{t}$ over that part of the dynamics between times $t-\tau$ and $t$, so that $\nu_{t, \tau}$ depends on $\mathcal{J}, \sigma^{0}$ and $\omega$ (before time $t-\tau$ ). 
Because detailed balance is satisfied by the dynamics, we expect that after a sufficiently long time $t$, the probability assigned by the dynamical measure $\nu_{t}(\sigma)$ (or by $\nu_{t, \tau}$ with both $t$ and $\tau$ large) to a given spin configuration $\sigma^{(L)}$ within $\Lambda_{L}$ will approach that assigned to that same configuration by some Gibbs state $\rho_{\mathcal{J}, T}$, and that this will be true for any $L$ and $\sigma^{(L)}$ (of course, how long one has to wait before this occurs will depend on $L$ ). Although this may be surprising at first, especially in light of assertions that equilibrium states are of little relevance for the nonequilibrium dynamics of infinite systems, it is to be expected if the common conjecture holds for Glauber dynamics (even in infinite volume) that at positive temperature only Gibbs states (where the probability of appearance of spin configurations are given by the Boltzmann distribution) are stationary. Note that the $\rho_{\mathcal{J}, T}$ in this discussion need not be a pure state.

\subsection{Equilibration and nonequilibration}

Now we can address the question of what it means for the system to evolve into (or within) a specific pure state. Since this involves some sort of equilibration, we need to address first the broader question of what equilibration means in an infinite system. This has been subject to various interpretations. A common viewpoint [49] is that infinite systems (following a deep quench, say) never reach equilibrium in any finite time; for example, in the homogeneous ferromagnet domains of positive and negative magnetization increase with time but are never infinite on any finite timescale. This is of course true, but we do not find it to be a useful way of looking at equilibration considering that equilibrium states are really a local concept (see, e.g., [50]).

Instead, we propose the following [51]: if for any finite region $\Lambda_{L}$ there exists a time $t_{L}^{*}<\infty$ after which the distribution $\nu_{t}$ (or $\nu_{t, \tau}$ ), restricted to $\Lambda_{L}$, is (approximately) the same as some pure state $\alpha$, then we say that the system has equilibrated in finite time. (It doesn't matter that the equilibration time depends on the region size.) So, in the case of the ferromagnet, our definition implies that an infinite ferromagnet equilibrates if, for any region of any size, domain walls (between positive and negative magnetization) cease to move across the region after some finite time (depending on the region).

Is this definition trivial? No, because it may be that such "local equilibration" does not occur. As a specific example, it doesn't occur for $2 D$ ferromagnets at low $T$; it can be rigorously proved that, following a deep quench, for any finite region, domain walls continually sweep across it (presumably at increasingly widely spaced time intervals) for all time [52]. (We don't know for sure what happens above two dimensions, but we conjecture, based on numerical work of Stauffer [53], that this "local nonequilibration" holds for the 
homogeneous ferromagnet up to four dimensions while at five dimensions and above local equilibration occurs.)

We will discuss local nonequilibration and its consequences more in Sec. 4. For now, however, we return to the question of pure states. If local equilibration has occurred and the dynamical distribution $\nu_{t}$ (or $\nu_{t, \tau}$ ) has approached a pure state $\alpha$, then the system has settled into that pure state. In that case the entire (infinite) system settles into $\alpha$; it cannot be that different regions have settled into different pure states (e.g., the positive and negative magnetization states in the homogeneous ferromagnet). But if local equilibration does not occur, it should still be true that (after some finite time depending on the region) any finite region will approximate a pure state (in the sense described above) at most times (the exceptional times being when a domain wall between different pure states sweeps across the region). The details behind this assertion can be found in Sec. 2 of [51].

In the case of local equilibration, the pure state $\alpha$ that the region $\Lambda_{L}$ has settled into at time $t$ should depend on $\mathcal{J}, \sigma^{0}$, and $\omega$. It is for that reason the the theorem discussed in the next section comes as a surprise, and has some far-reaching consequences.

\section{Basins of attraction of pure states form a set of mea- sure zero}

We have seen that the problem of equilibration at positive temperature boils down to the question of whether, on every fixed (and arbitrarily large) lengthscale $L$ the dynamical measure $\nu_{t}$ (more precisely $\nu_{t, \tau}$ for some $\tau(t) \rightarrow \infty$ with $t$ ) settles down to a pure state Gibbs measure $\rho^{\alpha}$ that is independent of time (after a timescale depending on $L$ ). Even if this doesn't occur, at most times larger than some $t_{L}^{*}$, the dynamical measure $\nu_{t}$ (more precisely $\left.\nu_{t, \tau(t)}\right)$ will approximate a pure state $\alpha(t)$; in this case, at presumably widely spaced times a domain wall (here to be thought of as the boundary separating two distinct pure states) sweeps across $\Lambda_{L}$, changing the pure state seen within the volume. Even so, if one simply chooses a fixed, arbitrary time much larger than $t_{L}^{*}$, with high probability the finite volume $\Lambda_{L}$ will be found inside some thermodynamic pure state.

This naturally raises the question: how does the system evolve, and what determines its long-time dynamical evolution? A standard viewpoint is that the system should evolve into some pure state, depending on initial conditions, within which it remains forever after. How

might this happen? Perhaps some part of the system finds a pure state before other parts (because it was fortuitously close to one to begin with), and this region then grows so that eventually any part of the system can be found in that same pure state. If other parts of 
the system fall into different pure states, then upon their boundaries meeting one such state, perhaps the one in the larger volume, would presumably "win".

Of course, if there is only one pure state governing the equilibrium thermodynamics (such as, e.g., the paramagnetic state in the uniform ferromagnet above $T_{c}$ ), then a simple version of something like this happens, and the system does settle into that pure state. However, if there is more than one pure state, whether two, ten, or an infinite number, then the scenario described above never happens for $\nu_{t}$. This claim follows from the following theorem:

Theorem 1 [51]: Given some $\mathcal{J}$ and $T>0$, assume that for almost every $\sigma^{0}, \nu_{t}$ converges to a limiting pure Gibbs state $\nu_{\infty}$ as $t \rightarrow \infty$. Then $\nu_{\infty}$ is the same pure state for almost every $\sigma^{0}$.

The proof of Theorem 1 is fairly short and appears in [51]. Here we are concerned only with its consequences. The theorem applies regardless of whether the system's equilibrium thermodynamics is governed by a single pure state (in which case the conclusion is trivial), or many.

In the latter case, of course we expect that the dynamical outcome should depend on the starting configuration. There are three possible ways to resolve this apparent contradiction:

1) $\nu_{\infty}$ is not a Gibbs state. This would violate the expected $T>0$ behavior discussed in Section 2 and hence we discard this possibility.

2) $\nu_{\infty}$ is a mixed Gibbs state (which may or may not depend on $\sigma^{0}$ ).

3) $\nu_{t}$ does not converge (to a single limit) as $t \rightarrow \infty$.

Let's consider the second and third possibilities in more detail. Possibility (2) implies that at (suitably large) time $t$, the dynamical measure $\nu_{t, \tau(t)}$ is approximately a pure state $\rho^{\alpha(t)}$, but that pure state depends not only on $\sigma^{0}$ (as expected) but also on $\omega$ (i.e., the dynamical realization between times 0 and $t-\tau)$. So, while the system might "land" in a pure state in the sense that $\alpha(t)$ converges to some $\alpha\left(\sigma^{0}, \omega\right)$, the limiting pure state is almost never determined solely by $\sigma^{0}$.

But this has a strong consequence for the geometry of the state space structure. The basin of attraction of a pure state $\bar{\alpha}$ is the set of configurations $\sigma^{0}$ such that $\alpha\left(\sigma^{0}, \omega\right)=\bar{\alpha}$ for almost every $\omega$ (see Ref. [54] for related discussions). Therefore, if many pure states exist (and the conclusion of Theorem 1 is not valid), then the union of all their basins of attraction must form a set of measure zero in the space of $\sigma^{0}$ 's; i.e., the configuration space resulting from a deep quench is all "boundary" in the sense that almost every initial configuration could land in one of several (or many) pure states depending on the realization of the dynamics (if it lands at all).

This result is perhaps counterintuitive. In the Introduction, it was noted that the context in which an experiment is done is crucial to the interpretation of statements relating 
equilibrium thermodynamic structure to nonequilibrium dynamical behavior. This example provides an important illustration of this. Because the quench is from a very high (formally, infinite) to a low temperature, the relevant configuration space that the system must explore (at least for small time $t$ ) is effectively the one prevailing at high temperatures. However, the pure state basins that the system evolves to are those relevant to the (low) temperature that determines the dynamical rules. Looked at in this way, it may not seem quite so strange (in fact, it seems quite natural) that the pure states form a set of measure zero in the configuration space. But then this also illustrates that any statements relating or contrasting equilibrium thermodynamics with nonequilibrium dynamics cannot in general be made independently of the dynamical process under consideration.

We note finally that Theorem 1 may be relevant to damage spreading [55, 56, 57], where one asks whether the damage (i.e., discrepancy) between $\sigma^{t}$ and $\sigma^{\prime t}$ (with a single $\omega$ ) grows as $t \rightarrow \infty$. Theorem 1 suggests that if damage spreading occurs, then $\nu_{t}$ doesn't converge to a single pure state (e.g., it might converge to a mixed state, as above).

\section{Effect of pure states on nonequilibrium dynamics}

Before discussing possibility (3), let us consider the physical picture implied by Theorem 1 . Roughly speaking, some time after an initial quench the system will form domains, whose average size increases with time, corresponding to the different pure states. This scenario has been analyzed for the two-state droplet picture [41, 58, 59]. It is also a well-known scenario for coarsening in a ferromagnet following a deep quench [49]. (Of course, in contrast to the spin glass case, one does know how to prepare a ferromagnet in a pure state; for a general discussion, see Ref. [60].)

But if possibility (3) holds, then even after a region has settled into a pure state, it remains "alive" dynamically: the pure state in that region will eventually change. In Sec. 2.3, we defined system equilibration after a finite time in terms of local equilibration: for any region $\Lambda_{L}$, there exists a time $t_{L}$ after which domain walls cease to move across the region. Possibility (3) would then correspond [51] to local non-equilibration (LNE) on any finite lengthscale. (We note that Possibility (2) could occur either with local equilibration or non-equilibration.)

So LNE means that in any fixed finite region, the system never settles down into a pure state. Domain walls do not simply move farther from the region as time progresses, but continually return and sweep across it, changing the state within. If LNE occurs in the spin glass, it would force us to revise the usual dynamical definition [1] of the EA order parameter. It could also mean that, for infinite systems, time averages and Gibbs averages could agree, 
despite the presence of many pure states.

We will return to this in Sec. 6 after investigating LNE in more detail by means of the next theorem, which applies to both homogeneous and disordered systems and ties together equilibrium pure state structure with nonequilibrium dynamics.

Theorem 2. [51] Let $\mathcal{N}$ be the number of pure states in the EA model for fixed $T$ and $d$, and suppose that $T$ and $d$ are such that all pure states have nonzero EA order parameter (implying that $\mathcal{N}$, which is the same for almost every $\mathcal{J}$, cannot be 1 ). If $\mathcal{N}$ is countable (including a countable infinity), then LNE occurs.

An immediate consequence of Theorem 2 is that if LNE does not occur (and the limiting pure states have nonzero $q_{E A}$ ), then there must be an uncountable number of pure states. Furthermore, the proof of Theorem 2 (see [51]), which is based on overlaps, shows that almost every pair of these pure states has overlap equal to zero. This shows that, as claimed in the Introduction, nonequilibrium dynamics can provide important information on the structure of equilibrium pure states, and vice-versa.

It also suggests a dynamical test of the two-state picture: search for LNE in the dynamical measure $\nu_{t}$ or $\nu_{t, \tau}$. If LNE does not occur, then the two-state picture has been ruled out there must be an uncountable number of pure states with almost all pairs having overlap zero (consistent with the results of Ref. [44]). If LNE does occur then neither the two-state nor the many-state pictures have been ruled out. (It is not very clear how one might go about observing LNE in a spin glass, where, unlike the ferromagnet, one doesn't know what a domain wall looks like. For a discussion of how this might be accomplished, see [51].)

Theorem 2 also implies that LNE occurs at small positive temperature in the $2 D$ uniform Ising ferromagnet and the random Ising ferromagnet for $d<5$. In the former case this result was extended to zero temperature (using different arguments) in [52. It was also shown there that for many systems (e.g., spin glasses and random ferromagnets where the common distribution of the $J_{x y}$ 's is continuous) that $\sigma^{t}$ does converge to some limit at $T=0$. (There are also systems, such as the $\pm J$ spin glass on the square lattice at $T=0$, where some spins flip only finitely many times and some spins flip infinitely often [61].) In light of these results, we restrict the term LNE to $T>0$, since in the zero-temperature situations where $\sigma^{t}$ converges, the limit configuration is typically only metastable rather than a ground state and so equilibration has not really occurred. In these systems one can define a dynamical order parameter, related to the autocorrelation, that does not decay to zero. 


\section{More about local non-equilibration}

To further clarify the discussion of LNE, consider the homogenous ferromagnet. At positive temperature, LNE is a phenomenon separate from the spontaneous formation of domains of the minority phase within the majority phase. The timescale for such a domain of size $L$ to form about the origin is exponential in (some power of) L. Similarly, for a finite system of size $L$, the entire system will randomly flip back and forth between the plus and minus phases on an exponential timescale. This, however, is not LNE, which takes place on much shorter time scales (presumably some power of $L$ ). LNE is not due to the spontaneous formation of one phase within another due to statistical fluctuations, but instead is due to domain walls sweeping into the region from far away. This contrast is even clearer at $T=0$, where the spontaneous formation of droplets described above cannot occur; but as already discussed, in the $2 D$ ferromagnet the phenomenon of domain walls forever sweeping across any finite region occurs even at zero temperature.

Since the existence of LNE for all $T<T_{c}$ in the $2 D$ Ising ferromagnet may seem surprising, we present a possible physical mechanism which may also shed light on LNE in general. The initial spin configuration has (with probability one) no infinite domains. As the configuration evolves, some domains shrink and others coalesce. So the origin should always be contained in a finite domain, whose size could usually be slowly decreasing, but sporadically would have a large change either by coalescing or because a domain wall passes through the origin and the identity of the domain changes. As a consequence we can arrive at the interesting situation where the mean scale of the domain containing the origin increases with $t$, even though, at a typical arbitrarily chosen time and for fixed $\sigma^{0}$ and $\omega$, its size would be decreasing. The dynamical behavior averaged over initial configurations and dynamical realizations is relatively straightforward, while for individual instances of both it is typically complex.

Summarizing, LNE is primarily the result of nonequilibrium domain wall motion driven by mean curvature combined with the complex domain structure resulting from the original quench. It is also consistent with phase separation (as would be expected from equilibrium roughening arguments).

\section{Dynamical vs. Boltzmann averages}

It was noted above that the presence of LNE might imply that some standard assumptions regarding broken ergodicity can fail. Suppose that LNE occurs because only a single pair of pure states is present, such as in the $2 D$ ferromagnet below $T_{c}$. In such a case, would 
a long time average of the magnetization in a finite region give zero? The answer is: not necessarily. It could be that, after long times, the system has spent roughly equal amounts of time in both states, in which case the usual time average [1] would indeed approach zero. But it could also happen that, after almost any long time, the system has spent significantly more of its life in one or the other state (which itself would change with the observational timescale). In other words (still using the example of a two-state system), at any long time the weights of the two states, as defined by a dynamical measure involving a fixed $\omega$ and an average over uniformly spaced times, could be different from $1 / 2$, and could even change with time (as discussed in the next section). This is analogous to an equilibrium phenomenon discovered by Külske [62, 63]. To get a zero average in this situation one would need to average over a sparse sequence of increasingly separated times.

\subsection{Chaotic Time Dependence}

We noted earlier that LNE can occur in the context of either possibility (2) (the averaged dynamical measure $\nu_{t}$ has a limit, which is a mixed state) or (3) ( $\nu_{t}$ does not converge). LNE must occur if possibility (3) holds, but may or may not occur if possibility (2) holds. We now explore further the distinctions between these two cases.

There are two ways in which possibility (2) can occur. As described earlier, for any fixed region $\Lambda_{L}$ the measure $\nu_{t, \tau(t)}$ can settle into a pure state for almost all $\sigma^{0}$ and $\omega$, but the pure state depends on the dynamics as well as the initial state. But a second possibility, that has not yet been discussed, is that $\nu_{t, \tau(t)}$ never settles down to a single pure state: the system is usually in a pure state $\alpha(t)$ locally, but the pure state forever changes. Nevertheless, $\nu_{t}$, the full average over the dynamics, still yields a single limit. This is to be contrasted with possibility (3), where even the fully averaged measure $\nu_{t}$ never settles down.

Again we use the illustration of the $2 D$ homogeneous ferromagnet to clarify these statements. Below $T_{c}$, we know LNE occurs by Theorem 2. Suppose furthermore that it occurs through possibility (2). Then, for fixed $\sigma^{0}$ and at a fixed large time, for approximately half of the dynamical realizations, a region of fixed lengthscale $L$ surrounding the origin is in the up state (the pure state $\rho^{+}$), and for most of the other half the same region is in the down state (the pure Gibbs state $\rho^{-}$), and this one-to-one ratio remains essentially fixed after some timescale depending on $L$. Then as $t \rightarrow \infty, \nu_{t} \rightarrow \bar{\rho}$, where $\bar{\rho}$ is the mixed Gibbs state $(1 / 2) \rho^{+}+(1 / 2) \rho^{-}$. Nevertheless, in any given dynamical realization, the region (as described by $\nu_{t, \tau}$ ) never settles permanently into either $\rho^{+}$or $\rho^{-}$.

By contrast, if possibility (3) occurs, then even the fully averaged dynamical measure

$\nu_{t}$ forever changes. This could happen (again for fixed $\sigma^{0}$ ) if the random dynamics fails 
to sufficiently "mix" the states (in which case one has, given $\sigma^{0}$, some amount of predictive power for determining from $\sigma^{0}$ the likely state of the system in the region for some arbitrarily large times $t$ ). This is conceivable because even though $\sigma^{0}$ is globally unbiased between the plus and minus states, it does have fluctuations in favor of one or the other state of order $\sqrt{L^{2}}$ on lengthscale $L$; with $L$ taken as an appropriate power of $t(L)$, these fluctuations could (partially) predict the sign of the phase at the origin at time $t(L)$. In possibility $(2)$ on the other hand, there is a greater capability of the random dynamics to "mix" the states which eventually destroys the predictive power contained in the fluctuations of the initial state.

So there are really two kinds of non-equilibration, corresponding either to LNE in the framework of possibility (2) ("weak LNE") or else to LNE resulting from the stronger possibility (3). Because $\nu_{t}$ evolves deterministically according to an appropriate master equation, its lack of a limit in possibility (3) corresponds conceptually to the usual notion of deterministic chaos and can thus legitimately be called chaotic time dependence (CTD) [51, 64. If weak LNE occurs, this term is not appropriate because here the effect is due to the random dynamics.

\section{Nature vs. Nurture}

The presence of two different ways the system can fail to equilibrate - weak LNE vs. CTD - leads to an interesting issue of predictably: if weak LNE occurs, the configuration $\sigma^{t}$ for large $t$ is determined essentially by the dynamics, and the initial configuration provides little predictive capability in determining the state of any particular spin at a very large time. If CTD occurs, on the other hand, then some predictive power from the initial configuration remains at arbitrarily large times. This "nature vs. nurture" competition provides an interesting set of problems for future study.

Can we determine which of these possibilities occurs for selected systems? One simple case where the extent of predictability can be precisely determined is the $1 D$ disordered ferromagnet (or spin glass) with a continuous coupling distribution (e.g., couplings chosen uniformly from $[0,1]$ for the ferromagnet and from the Gaussian distribution for a spin glass). We define (for general dimension) a dynamical order parameter $q^{t}$ as $[52]$

$$
q^{t}=\lim _{L \rightarrow \infty}(2 L+1)^{-d} \sum_{x \in \Lambda_{L}}\left(\left\langle\sigma_{x}\right\rangle_{t}\right)^{2}=\mathbf{E}_{\mathcal{J}, \sigma^{0}}\left(\left\langle\sigma_{y}\right\rangle_{t}^{2}\right) .
$$

In this formula, dynamical averages (i.e., with respect to the distribution $\nu_{t}$ over dynamical realizations $\omega$ ) of $\sigma^{t}$ (with fixed $\mathcal{J}, \sigma^{0}$ ) are denoted by $\langle\cdot\rangle_{t}$ and $y$ is any fixed site, e.g., the

origin; the remaining averages, over $\mathcal{J}$ and $\sigma^{0}$, are denoted by $\mathbf{E}_{\mathcal{J}, \sigma^{0}}$. The equivalence of the two formulas for $q^{t}$ follows from translation-ergodicity; see [52] for details. 
If the infinite-time limit of $q^{t}$ exists, we define $q_{D}=\lim _{t \rightarrow \infty} q^{t}$. The order parameter $q_{D}$ measures the extent to which $\sigma^{\infty}$ is determined by $\sigma^{0}$ rather than by $\omega$; it is a dynamical analogue to the usual Edwards-Anderson order parameter. Of course, $q^{0}=1$ because $\sigma^{0}$ is completely determined by $\sigma^{0}$, while a value $q_{D}=0$ would mean that for every $x,\left\langle\sigma_{x}\right\rangle_{t} \rightarrow 0$ so that $\sigma^{0}$ yields no information about $\sigma^{t}$ as $t \rightarrow \infty$. The following theorem provides an exact determination of $q_{D}$ for the disordered $1 D$ systems introduced above:

Theorem 3. [52] For the $d=1$ homogeneous ferromagnet at zero temperature, $\sigma_{x}^{\infty}$ does not exist (i.e., $\sigma_{x}^{t}$ changes infinitely many times as $t \rightarrow \infty$ ) for almost every $\sigma^{0}$ and $\omega$ and every $x$. For the corresponding one-dimensional disordered model (feromagnet or spin glass) with continuous coupling distribution, $\sigma_{x}^{\infty}$ does exist for almost every $\mathcal{J}, \sigma^{0}$, and $\omega$ and every $x$; furthermore $q_{D}=1 / 2$.

The value $q_{D}=1 / 2$ is a reflection of the fact that, for almost every $\mathcal{J}$ and $\sigma^{0}$, precisely half of the $x$ 's in $\mathbf{Z}$ have $\sigma_{x}^{\infty}$ completely determined by $\sigma^{0}$ with the other $\sigma_{x}^{\infty}$ 's completely undetermined by $\sigma^{0}$. For the homogeneous one-dimensional ferromagnet, it is not hard to show (see, e.g., [64]) that although $\sigma^{\infty}$ does not exist, $\langle\cdot\rangle_{\infty}$ does exist for almost all $\sigma^{0}$, and that $q_{D}=0$.

What about higher-dimensional systems? Here we mostly need to rely on numerical studies, at least for the present. In a recent paper [65], the homogeneous $2 D$ ferromagnet on a square lattice was studied at zero temperature. Numerical results from this study suggest that CTD might hold for the infinite lattice, in which case long-term predictability from information contained in the initial state would be present to some extent. We refer the interested reader to [65] for details.

\section{Summary}

In this review, we considered the dynamical evolution of a short-range Ising spin glass following a deep quench (although many of our results generalize to other systems). We presented several theorems [51] with somewhat surprising consequences, as follows. If the spin glass displays broken spin-flip symmetry (more precisely, has a nonzero EA order parameter), then equilibration in any local region depends crucially not only on the number of pure states but also their overlaps. Only when there exists an uncountable infinity of pure states, with almost every pair having zero overlap (i.e., the spin overlap distribution $P(q)$ is a $\delta$-function at zero), can the system equilibrate, falling into some pure state as $t \rightarrow \infty$. However, this is a necessary, not a sufficient condition.

A second consequence of these theorems is that the union of the basins of attraction of all 
pure states (again, if broken symmetry occurs) forms a set of measure zero in configuration space following a deep quench: almost every starting configuration is on a boundary between multiple pure state basins.

This has consequences not only for deep quenches but also for slow cooling. Once again the ferromagnet provides an instructive example. The general applicability of our arguments implies that the same result holds for ferromagnets (either homogeneous or disordered) following a deep quench. But if one cools slowly instead, then it's easy to prepare the system in one of the two translationally invariant pure states, which are well understood and characterized: the positive and negative magnetization states.

But the spin glass could present a different story under slow cooling, even for small temperature changes. If the chaotic temperature dependence predicted in some theories [42, 43 occurs, then the pure state structure of a spin glass (with fixed $\mathcal{J}$ ) changes chaotically on lengthscales larger than some $L^{*}(\Delta T)$ when the system undergoes a change in temperature $\Delta T$. The dynamical effect of such a change may then be similar to that of a deep quench. The well-known difficulty in equilibrating spin glasses may therefore be a consequence of this effect, with long relaxation times arising from small domain sizes and slow (possibly due to pinning) motion of domain walls.

More generally, we have argued against a common viewpoint that pure state multiplicity is irrelevant to the dynamics of infinite (or very large) systems on finite timescales. In many situations, a system will not spend all of its time in a single pure state, even locally. Because of this, it is also not necessarily true that "absolutely broken ergodicity" — i.e., the presence of more than one pure state separated by infinite barriers - implies that time averages and Boltzmann averages must disagree (or equivalently, that the $\operatorname{limits} N \rightarrow \infty$ and $t \rightarrow \infty$ cannot commute).

Finally, we discussed the effect of initial conditions on the future spin configuration of a spin system, in the context of its predictability: to what extent is the evolution determined by the starting configuration, and how much depends on the dynamics? This "nature vs. nurture" problem can be solved exactly for $1 D$ random ferromagnets and spin glasses [52], and was studied numerically for the $2 D$ homogeneous ferromagnet on the square lattice [65]. The problem is equivalent to determining whether a weak form of local non-equilibration occurs (which favors "nurture") or whether a stronger chaotic time dependence occurs (which favors "nature"). Which of these occurs for particular systems remains an open problem.

Acknowledgments. This work was partially supported by the National Science Foundation under grant DMS-0604869. We thank Joerg Rottler, Malcolm Kennett, and Philip Stamp, guest editors of the special issue entitled "Classical and Quantum Glasses" of the Journal of Physics: Condensed Matter, for inviting us to contribute an article. 


\section{References}

[1] K. Binder and A. P. Young. Spin glasses: experimental facts, theoretical concepts, and open questions. Rev. Mod. Phys., 58:801-976, 1986.

[2] P. Bouchaud, L. Cugliandolo, J. Kurchan, and M. Mézard. Out of equilibrium dynamics in spin-glasses and other glassy systems. In A. P. Young, editor, Spin Glasses and Random Fields, pages 161-223. World Scientific, Singapore, 1998.

[3] P. Refrigier, E. Vincent, J. Hamman, and M. Ocio. Ageing phenomena in a spin glass: effect of temperature changes below $T_{g}$. J. Phys. (Paris), 48:1533-1539, 1987.

[4] G. J. M. Koper and H. J. Hilhorst. A domain theory for linear and nonlinear aging effects in spin glasses. J. Phys. (Paris), 49:429-443, 1988.

[5] P. Sibani and K.-H. Hoffmann. Hierarchical models for aging and relaxation of spin glasses. Phys. Rev. Lett., 63:2853-2856, 1989.

[6] K.-H. Hoffmann and P. Sibani. Relaxation and aging in spin glasses and other complex systems. Z. Phys. B, 80:429-438, 1990.

[7] P. Svedlindh, K. Gunnarson, J.-O. Andersson, H. A. Katori, and A. Ito. Time-dependent AC susceptibility in spin glasses. Phys. Rev. B, 46:13867-13873, 1992.

[8] J. P. Bouchaud. Weak ergodicity breaking and aging in disordered systems. J. Phys. I, 2:1705-1713, 1992.

[9] F. Lefloch, J. Hamann, M. Ocio, and E. Vincent. Can aging phenomena discriminate between the droplet model and a hierarchical description in spin glasses? Europhys. Lett., 18:647-652, 1992.

[10] H. Rieger. Nonequilibrium dynamics and aging in the three-dimensional Ising spin-glass model. J. Phys. A, 26:L615-L621, 1993.

[11] S. Franz and M. Mézard. On mean field glassy dynamics out of equilibrium. Physica A, 210:48-72, 1994.

[12] E. Vincent, J. P. Bouchaud, D. S. Dean, and J. Hamann. Aging in spin glasses as a random walk: effect of a magnetic field. Phys. Rev. B, 52:1050-1060, 1995.

[13] M. Lederman, R. Orbach, J. M. Hamann, M. Ocio, and E. Vincent. Dynamics in spin glasses. Phys. Rev. B, 44:7403-7412, 1988.

[14] J. M. Hammann, M. Lederman, M. Ocio, R. Orbach, and E. Vincent. Spin-glass dynamics. Relation between theory and experiment: a beginning. Physica A, 185:278-294, 1992. 
[15] Y. G. Joh, R. Orbach, and J. Hamann. Spin glass dynamics under a change in a magnetic field. Phys. Rev. Lett., 77:4648-4651, 1996.

[16] E. Vincent, J. Hammann, M. Ocio, J.-P. Bouchaud, and L. Cugliandolo. Slow dynamics and aging in spin glasses. In M. Rubi, editor, Complex Behavior of Glassy Systems, pages 184-219. Springer-Verlag Lecture Notes in Physics, v. 492, Berlin, 1997.

[17] K. Jonason, E. Vincent, J. Hammann, J.-P. Bouchaud, and P. Nordblad. Memory and chaos effects in spin glasses. Phys. Rev. Lett., 81:3243-3246, 1998.

[18] B. Rinn, P. Maass, and J.-P. Bouchaud. Multiple scaling regimes in simple aging models. Phys. Rev. Lett., 84:5403-5406, 2000.

[19] F. Ricci-Tersenghi and R. Zecchina. Glassy dynamics near zero temperature. Phys. Rev. E, 62:R7567-R7570, 2000.

[20] E. Vincent, V. Dupuis, M. Alba, J. Hamann, and J.-P. Bouchaud. Aging phenomena in spin glass and ferromagnetic phases: domain growth and wall dynamics. Europhys. Lett., 50:674680,2000 .

[21] A. T. Ogielski. Dynamics of three-dimensional spin glasses in thermal equilibrium. Phys. Rev. $B, 32: 7384-7398,1985$.

[22] A. T. Ogielski and I. Morgenstern. Critical behavior of the three-dimensional Ising spin-glass model. Phys. Rev. Lett., 54:928-931, 1985.

[23] J. D. Reger, R. N. Bhatt, and A. P. Young. Monte Carlo study of the order-parameter distribution in the four-dimensional Ising spin glass. Phys. Rev. Lett., 64:1859-1862, 1990.

[24] N. Kawashima and A. P. Young. Phase transition in the three-dimensional $\pm J$ Ising spin glass. Phys. Rev. B, 53:R484-R487, 1996.

[25] M. Palassini and A. P. Young. Evidence for a trivial ground-state structure in the twodimensional Ising spin glass. Phys. Rev. B, 60:R9919-R9922, 1999.

[26] M. Palassini and A. P. Young. Triviality of the ground state structure in Ising spin glasses. Phys. Rev. Lett., 83:5126-5129, 1999.

[27] A. A. Middleton. Numerical investigation of the thermodynamic limit for ground states in models with quenched disorder. Phys. Rev. Lett., 83:1672-1675, 1999.

[28] A. A. Middleton. Energetics and geometry of excitations in random systems. Phys. Rev. B, 63:060202, 2000.

[29] A. K. Hartmann. How to evaluate ground-state landscapes of spin glasses thermodynamically correctly. Eur. Phys. J. B, 13:539-545, 2000. 
[30] F. Krzakala and O. C. Martin. Spin and link overlaps in three-dimensional spin glasses. Phys. Rev. Lett., 85:3013-3016, 2000.

[31] M. Palassini and A. P. Young. Nature of the spin glass state. Phys. Rev. Lett., 85:3017-3020, 2000 .

[32] J. Houdayer, F. Krzakala, and O. C. Martin. Large-scale low-energy excitations in 3-d spin glasses. Replica symmetry breaking and characterization in position space. Eur. Phys. B, 18:467-477, 2000.

[33] E. Marinari and G. Parisi. Effects of changing the boundary conditions on the ground state of Ising spin glasses. Phys. Rev. B, 62:11677-11685, 2000.

[34] E. Marinari and G. Parisi. Effects of a bulk perturbation on the ground state of $3 D$ Ising spin glasses. Phys. Rev. Lett., 86:3887-3890, 2001.

[35] G. Hed, A. P. Young, and E. Domany. Lack of ultrametricity in the low-temperature phase of three-dimensional ising spin glasses. Phys. Rev. Lett., 92:157201, 2004.

[36] M. Mézard, G. Parisi, and M. A. Virasoro, editors. Spin Glass Theory and Beyond. World Scientific, Singapore, 1987.

[37] E. Marinari, G. Parisi, F. Ricci-Tersenghi, J. J. Ruiz-Lorenzo, and F. Zuliani. Replica symmetry breaking in spin glasses: Theoretical foundations and numerical evidences. J. Stat. Phys., 98:973-1047, 2000.

[38] W. L. McMillan. Scaling theory of Ising spin glasses. J. Phys. C, 17:3179-3187, 1984.

[39] A. J. Bray and M. A. Moore. Critical behavior of the three-dimensional Ising spin glass. Phys. Rev. B, 31:631-633, 1985.

[40] D. S. Fisher and D. A. Huse. Ordered phase of short-range Ising spin-glasses. Phys. Rev. Lett., 56:1601-1604, 1986.

[41] D. S. Fisher and D. A. Huse. Nonequilibrium dynamics of spin glasses. Phys. Rev. B, 38:373$385,1988$.

[42] D. S. Fisher and D. A. Huse. Equilibrium behavior of the spin-glass ordered phase. Phys. Rev. $B, 38: 386-411,1988$.

[43] A. J. Bray and M. A. Moore. Chaotic nature of the spin-glass phase. Phys. Rev. Lett., 58:57-60, 1987.

[44] C. M. Newman and D. L. Stein. Metastate approach to thermodynamic chaos. Phys. Rev. E, 55:5194-5211, 1997. 
[45] C. M. Newman and D. L. Stein. Simplicity of state and overlap structure in finite-volume realistic spin glasses. Phys. Rev. E, 57:1356-1366, 1998.

[46] C. M. Newman and D. L. Stein. Topical Review: Ordering and broken symmetry in shortranged spin glasses. J. Phys.: Cond. Mat., 15:R1319 - R1364, 2003.

[47] C. M. Newman and D. L. Stein. Metastable states in spin glasses and disordered ferromagnets. Phys. Rev. E, 60:5244-5260, 1999.

[48] S. Edwards and P. W. Anderson. Theory of spin glasses. J. Phys. F, 5:965-974, 1975.

[49] A. J. Bray. Theory of phase-ordering kinetics. Adv. Phys., 43:357-459, 1994.

[50] C. M. Newman and D. L. Stein. Local vs. global variables for spin glasses. In E. Bolthausen and A. Bovier, editors, Spin Glass Theory, pages 145-158. Springer, Berlin, 2006.

[51] C. M. Newman and D. L. Stein. Equilibrium pure states and nonequilibrium chaos. J. Stat. Phys., 94:709-722, 1999.

[52] S. Nanda, C. M. Newman, and D. L. Stein. Dynamics of Ising spin systems at zero temperature. In R. Minlos, S. Shlosman, and Y. Suhov, editors, On Dobrushin's Way (from Probability Theory to Statistical Physics), pages 183-194. Amer. Math. Soc. Transl. (2) 198, 2000.

[53] D. Stauffer. Ising spinodal decomposition at $T=0$ in one to five dimensions. J. Phys. A, 27:5029-5032, 1994.

[54] A. C. D. van Enter and J. L. van Hemmen. Statistical-mechanical formalism for spin-glasses. Phys. Rev. A, 29:355-365, 1984.

[55] F. Bagnoli. On damage-spreading transitions. J. Stat. Phys., 85:151-164, 1996.

[56] P. Grassberger. Are damage spreading transitions generically in the universality class of directed percolation? J. Stat. Phys., 79:13-23, 1995.

[57] N. Jan and T. S. Ray. "Damage" in the low-temperature phase of the $\pm J$ spin glass in two to six dimensions. J. Stat. Phys., 75:1197-1204, 1994.

[58] G. J. M. Koper and H. J. Hilhorst. A domain theory for linear and nonlinear aging effects in spin glasses. J. Phys. (Paris), 49:429-443, 1988.

[59] M. J. Thill and H. J. Hilhorst. Theory of the critical state of low-dimensional spin glass. J. Phys. I, 6:67-95, 1996.

[60] R. G. Palmer. Broken ergodicity. Adv. Phys., 31:669-735, 1982.

[61] A. Gandolfi, C.M. Newman, and D.L. Stein. Zero temperature dynamics of $\pm J$ spin glasses and related Ising models. Commun. Math. Phys., 214:373-387, 2000. 
[62] C. Külske. Limiting behavior in random Gibbs measures: Metastates in some disordered mean field models. In A. Bovier and P. Picco, editors, Mathematics of Spin Glasses and Neural Networks, pages 151-160. Birkhauser, Boston, 1998.

[63] C. Külske. Metastates in disordered mean-field models II: The superstates. J. Stat. Phys., 91:155-176, 1998.

[64] L. R. G. Fontes, M. Isopi, and C. M. Newman. Random walks with strongly inhomogeneous rates and singular diffusions: convergence, localization and aging in one dimension. Ann. Probab., 30:579-604, 2002.

[65] P. M. C. de Oliveira, C. M. Newman, V. Sidoravicious, and D. L. Stein. Ising ferromagnet: Zero-temperature dynamical evolution. J. Phys. A, 39:6841-6849, 2006. 